\title{
The nature of $Z_{b}$ states from a combined analysis of $\Upsilon(5 S) \rightarrow h_{b}(m P) \pi^{+} \pi^{-}$and $\Upsilon(5 S) \rightarrow B^{(*)} \bar{B}^{(*)} \pi$
}

\author{
Wen-Sheng Huo ${ }^{1}$, Guo-Ying Chen ${ }^{1,2, a}$ \\ ${ }^{1}$ Department of Physics, Xinjiang University, Ürümqi 830046, China \\ ${ }^{2}$ State Key Laboratory of Theoretical Physics, Institute of Theoretical Physics, Chinese Academy of Sciences, Beijing 100190, China
}

Received: 5 November 2015 / Accepted: 11 March 2016 / Published online: 29 March 2016

(C) The Author(s) 2016. This article is published with open access at Springerlink.com

\begin{abstract}
With a combined analysis of data on $\Upsilon(5 S) \rightarrow$ $h_{b}(1 P, 2 P) \pi^{+} \pi^{-}$and $\Upsilon(5 S) \rightarrow B^{(*)} \bar{B}^{(*)} \pi$ in an effective field theory approach, we determine resonance parameters of $Z_{b}$ states in two scenarios. In one scenario we assume that $Z_{b}$ states are pure molecular states, while in the other one we assume that $Z_{b}$ states contain compact components. We find that the present data favor that there should be some compact components inside $Z_{b}^{(\prime)}$ associated with the molecular components. By fitting the invariant mass spectra of $\Upsilon(5 S) \rightarrow h_{b}(1 P, 2 P) \pi^{+} \pi^{-}$and $\Upsilon(5 S) \rightarrow$ $B^{(*)} \bar{B}^{*} \pi$, we determine that the probability of finding the compact components in $Z_{b}$ states may be as large as about $40 \%$.
\end{abstract}

\section{Introduction}

Two charged bottomonium-like states $Z_{b}^{ \pm}(10610)$ and $Z_{b}^{ \pm}(10650)$-denoted as $Z_{b}$ and $Z_{b}^{\prime}$-were discovered by the Belle Collaboration in decays $\Upsilon(5 S) \rightarrow \Upsilon(n S) \pi^{+} \pi^{-}$ for $n=1,2$, or 3 and $\Upsilon(5 S) \rightarrow h_{b}(m P) \pi^{+} \pi^{-}$for $m=1$ or $2[1,2]$. The masses and decay widths averaged over the five channels are $m_{Z_{b}}=10607.2 \pm 2.0 \mathrm{MeV}$, $\Gamma_{Z_{b}}=18.4 \pm 2.4 \mathrm{MeV}$, and $m_{Z_{b}^{\prime}}=10652.2 \pm 1.5 \mathrm{MeV}$, $\Gamma_{Z_{b}^{\prime}}=11.5 \pm 2.2 \mathrm{MeV}$ [3]. The average masses are about 2 $\mathrm{MeV}$ above the thresholds of both $B^{*} \bar{B}$ and $B^{*} \bar{B}^{*}$. Recently, Belle [4] also reported the observation of these two $Z_{b}$ states in $\Upsilon(5 S) \rightarrow\left[B \bar{B}^{*}+c . c.\right] \pi$, and $\Upsilon(5 S) \rightarrow B^{*} \bar{B}^{*} \pi$. The discovery of the $Z_{b}$ states has inspired many interesting theoretical discussions. For example, it is suggested that these states can be molecular states of the $B \bar{B}^{*}+$ c.c. or $B^{*} \bar{B}^{*}$ meson pairs [5-12]. They are also proposed to be candidates of tetraquark states [13]. In Ref. [14,15] the threshold enhancements are considered to be caused by cusp effects.

\footnotetext{
a e-mail: chengy@pku.edu.cn
}

Although the masses of these $Z_{b}$ states determined from the experimental fits are slightly above the thresholds, one should note that the masses are extracted by the BreitWigner parametrization. As emphasized in $[9,12]$, if an $S$ wave shallow bound state exists below the threshold, the amplitude should not be parameterized in the Breit-Wigner form. Using the line shape for a pure bound state, Ref. [9] shows that the data on $\Upsilon(5 S) \rightarrow h_{b}(m P) \pi^{+} \pi^{-}$are consistent with the bound state nature of $Z_{b}^{(\prime)}$. Furthermore, the observed enhancements in $\Upsilon(5 S) \rightarrow\left[B \bar{B}^{*}+\right.$ c.c. $] \pi$ and $\Upsilon(5 S) \rightarrow B^{*} \bar{B}^{*} \pi$ by Belle are very close to the thresholds of the $B^{(*)} \bar{B}^{(*)}$ systems. It is also found that the masses of the $Z_{b}$ states can be below the corresponding thresholds if these masses are extracted from data on $\Upsilon(5 S) \rightarrow B^{(*)} \bar{B}^{(*)} \pi[4]$.

As an observational fact, $Z_{b}$ states and their analogs in the charmonium sector $Z_{c}$ (3900) [16-18], $Z_{c}$ (4020/4025) [19, 20], and also the famous $X(3872)$ appear to be strongly correlated to the thresholds of either $B^{(*)}$ or $D^{(*)}$ pairs. This feature makes it natural to interpret these states as molecules. However, as was pointed out in Ref. [21,22], it is difficult to understand the large production rates of these states in $B$-factories, e.g. X(3872), if these states are assumed to be loosely bound molecular states. In particular, the recent $\mathrm{LHCb}$ measurement of the ratio $R_{\psi \gamma}=$ $\frac{\mathcal{B}(X(3872) \rightarrow \psi(2 S) \gamma)}{\mathcal{B}(X(3872) \rightarrow J / \psi \gamma)}=2.46 \pm 0.64 \pm 0.29$ [23] seems not to support a pure $D^{* 0} \bar{D}^{0}$ molecular interpretation of $X(3872)$, since $R_{\psi \gamma}$ is predicted to be rather small for a pure $D^{* 0} \bar{D}^{0}$ molecule [24]. Meanwhile, a compact component inside such states can compromise both threshold phenomena and sizable production rates. It is shown in Ref. $[25,26]$ that the radiative decays of $X(3872)$ are not only sensitive to long-range parts but also to short-range parts of the wave function. The search for a hidden-beauty counterpart of $X$ (3872), which is usually denoted as $X_{b}$, is important for understanding the structure of $X(3872)$. An effective field 
theory study shows that if $X(3872)$ is a molecular bound state of $D^{* 0}$ and $\bar{D}^{0}$ mesons, the heavy-quark symmetry requires the existence of a molecular bound state $X_{b}$ of $B^{* 0} \bar{B}^{0}$ with mass of $10604 \mathrm{MeV}$ [27]. However, there is no significant signal of $X_{b}$ near the threshold of $B^{* 0} \bar{B}^{0}$ in $X_{b} \rightarrow \pi^{+} \pi^{-} \Upsilon(1 S)$ [28] and in $X_{b} \rightarrow \omega \Upsilon(1 S)$ [29]. Reference [30] suggests that $X_{b}$ may be close in mass to the bottomonium state $\chi_{b 1}(3 P)$ and mixes with it. Therefore, the experiments which reported observing $\chi_{b 1}(3 P)$ might have actually discovered $X_{b}$.

Obviously, more experimental data and theoretical development are required to clarify the nature of these near threshold states. In Ref. [31] an effective field theory (EFT) approach is proposed for the study of near threshold states (see also an independent study in Ref. [32]). In this framework the compositeness theorem can be incorporated with a determination of parameter $Z$, which is the probability of finding an elementary component in the bound state, and the nature of near threshold states can be described by the presence of both molecular and compact components in their wavefunctions.

The main purpose of this work is to study structure of $Z_{b}$ states by doing a combined analysis of data on $\Upsilon(5 S) \rightarrow h_{b}(m P) \pi^{+} \pi^{-}$and $\Upsilon(5 S) \rightarrow B^{(*)} \bar{B}^{(*)} \pi$ within EFT approach proposed in [31]. Our work is organized as follows: in Sect. 2, we recall the EFT approach proposed in Ref. [31]. In Sect. 3, we present the analysis of the $\Upsilon(5 S) \rightarrow h_{b}(m P) \pi^{+} \pi^{-}$transitions and in Sect. 4, the $\Upsilon(5 S) \rightarrow B^{(*)} \bar{B}^{(*)} \pi$. Our numerical results are presented in Sect. 5. Finally, a brief summary is given in Sect. 6.

\section{Compositeness theorem in EFT}

In Ref. [31], we have developed an EFT approach which incorporates Weinberg's compositeness theorem [33,34]. Here we recall some of the main points; more details can be found in Ref. [31]. Consider a bare state $|\mathcal{B}\rangle$ with bare mass $-B_{0}$ and coupling $g_{0}$ to the two-particle state, where the bare mass is defined relative to the two-particle threshold. The two particles have masses $m_{1}, m_{2}$, respectively. If $|\mathcal{B}\rangle$ is near the two-particle threshold, then the leading two-particle scattering amplitude can be obtained by summing the Feynman diagrams in Fig. 1. Near threshold, the momenta of these two particles are non-relativistic. Therefore, the loop integral in Fig. 1 can be done in the same way as that in Ref. [35,36]. The loop integral can be written as

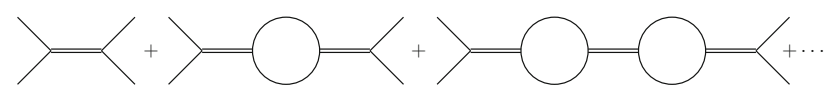

Fig. 1 Feynman diagrams for the two-particle scattering. The double lines denote the bare state

$$
\begin{aligned}
\mathcal{I}= & \int \frac{\mathrm{d}^{D} \ell}{(2 \pi)^{D}} \frac{i}{\ell^{0}-\vec{\ell}^{2} /\left(2 m_{1}\right)+i \epsilon} \\
& \cdot \frac{i}{E-\ell^{0}-\vec{\ell}^{2} /\left(2 m_{2}\right)+i \epsilon}, \\
= & \int \frac{\mathrm{d}^{D-1} \ell}{(2 \pi)^{D-1}} \frac{i}{E-\vec{\ell}^{2} /(2 \mu)+i \epsilon}, \\
= & -i 2 \mu \Gamma\left(\frac{3-D}{2}\right)(4 \pi)^{\frac{1-D}{2}}(-2 \mu E-i \epsilon)^{\frac{D-3}{2}},
\end{aligned}
$$

where $\mu$ is the reduced mass of the two particles, and $E$ is the kinematic energy of the two-particle system. Obviously, the above integral does not diverge in $D=4$. Using the minimal subtraction (MS) scheme which subtracts the $1 /(D-4)$ pole before taking the $D \rightarrow 4$ limit, one obtains

$\mathcal{I}=i \frac{\mu}{2 \pi}(-2 \mu E-i \epsilon)^{1 / 2}$.

It is interesting to note that with the MS scheme no counter term is needed in the renormalization. We then have the two body elastic scattering amplitude for Fig. 1:

$\mathcal{A}=-\frac{g_{0}^{2}}{E+B_{0}-g_{0}^{2} \frac{\mu}{2 \pi} \sqrt{-2 \mu E-i \epsilon}}$.

If a bound state exists, we have the following relations:

$g_{0}^{2}=g^{2} / Z, \quad B_{0}=\frac{2-Z}{Z} B, \quad g^{2}=\frac{2 \pi \sqrt{2 \mu B}}{\mu^{2}}(1-Z)$,

where $\mathrm{B}$ is the binding energy, and $Z$ is the probability of finding an elementary state in the physical bound state. Note that for the bound state, we mean a below threshold pole in the physical sheet. With Eq. (4), Eq. (3) can be re-expressed as

$\mathcal{A}=-\frac{g^{2}}{E+B+\tilde{\Sigma}(E)}$,

where

$\tilde{\Sigma}(E)=-g^{2}\left[\frac{\mu}{2 \pi} \sqrt{-2 \mu E-i \epsilon}+\frac{\mu \sqrt{2 \mu B}}{4 \pi B}(E-B)\right]$.

We can also express Eq. (5) in the form

$i \mathcal{A}=i g_{0} \cdot G(E) \cdot i g_{0}$,

where $G(E)$ is the complete propagator for the $S$-wave near the threshold state

$G(E)=\frac{i Z}{E+B+\tilde{\Sigma}(E)+i \Gamma / 2}$. 
We have added a constant width $\Gamma$ in the propagator, which can simulate the decay channels other than the bottom and anti-bottom mesons. From Eq. (7), one can find that the Feynman rule for the coupling between the near threshold state and its two-particle component is $i g_{0}$. Treating the binding momentum $\gamma=(2 \mu B)^{1 / 2}$ and the three-momentum of the two-particle state $p$ as small scales, i.e., $\gamma, p \sim \mathcal{O}(p)$, one can then find that the leading amplitude Eq. (5) is at the order of $\mathcal{O}\left(p^{-1}\right)$.

\section{$3 \Upsilon(5 S)$ decays to $h_{b}(1 P, 2 P) \pi^{+} \pi^{-}$}

In this section, we study the decay $\Upsilon(5 S) \rightarrow Z_{b}^{(\prime)} \pi \rightarrow$ $h_{b}(m P) \pi \pi$ in the EFT approach. Generally, in the decay $\Upsilon(5 S) \rightarrow Z_{b}^{(\prime)} \pi, Z_{b}$ states can be produced through both direct and indirect processes. In direct production processes, $Z_{b}$ states are produced directly via its compact component, while in indirect production processes a bottom and antibottom meson pair is produced first in the $\Upsilon(5 S)$ decay and then rescatters to $Z_{b}^{(\prime)}$. Similarly, the decay $Z_{b}^{(\prime)} \rightarrow h_{b}(m P) \pi$ can proceed through both direct and indirect processes. In direct decay, $Z_{b}$ states will decay to $h_{b}(m P) \pi$ directly. In indirect decay, $Z_{b}^{(\prime)}$ will first decay into a bottom and antibottom meson pair and then the meson pair rescatters into $h_{b}(m P) \pi$.

The three-momenta of heavy mesons in decay $\Upsilon(5 S) \rightarrow$ $Z_{b}^{(\prime)} \pi \rightarrow h_{b}(m P) \pi \pi$ are small compared with their masses. Therefore, these heavy mesons can be treated as nonrelativistic, and one can set up a power counting in terms of the small three-momentum $p$ [31,37-39]. From the power counting, one can find that if $Z_{b}^{(\prime)}$ contains a compact component, its production will be driven by this compact component [31] (see also Refs. [21,22]). In Fig. 2, we show Feynman diagrams where the $Z_{b}$ states are produced via compact components and decay through both direct and indirect processes.

If $Z_{b}^{(\prime)}$ is a pure molecular state, its production should go via indirect processes. The leading Feynman diagrams for indirect production of $Z_{b}^{(\prime)}$ are shown in Fig. 3. Note that there are two kinds of indirect production mechanisms for $Z_{b}$ states. In Fig. 3a, b, $\Upsilon(5 S)$ first decays to a bottom and anti-bottom meson pair and pion in the same vertex, then

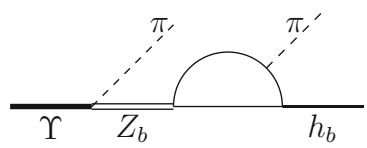

(a)

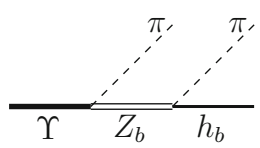

(b)
Fig. 2 Feynman diagrams for $\Upsilon(5 S) \rightarrow Z_{b}^{(\prime)} \pi \rightarrow h_{b}(m P) \pi \pi$, where the $Z_{b}$ states are produced in direct production processes. Solid lines in the loop represent bottom and anti-bottom mesons

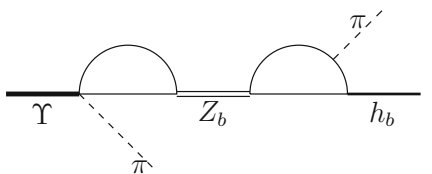

(a)

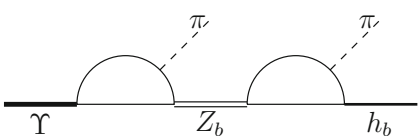

(c)

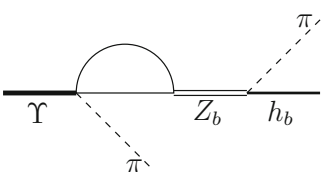

(b)

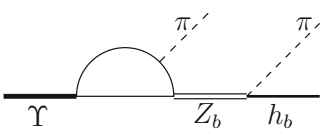

(d)
Fig. 3 Feynman diagrams for $\Upsilon(5 S) \rightarrow Z_{b}^{(\prime)} \pi \rightarrow h_{b}(m P) \pi \pi$, where the $Z_{b}$ states are produced in indirect production processes. Solid lines in the loops represent bottom and anti-bottom mesons

the bottom and anti-bottom meson pair rescatters to $Z_{b}^{(\prime)}$. While in Fig. 3c, d, $\Upsilon(5 S)$ first decays to a bottom and antibottom meson pair, and after emitting one pion, the bottom and anti-bottom meson pair rescatters to $Z_{b}^{(\prime)}$. It is shown in Ref. [12] that both mechanisms contribute at leading order for the indirect production of $Z_{b}$ states.

As we are only interested in low energy physics, it is convenient to collect $B$ mesons in a $2 \times 2$ matrix $[40,41]$,

$$
\begin{aligned}
& H_{a}=\vec{P}_{a}^{*} \cdot \vec{\sigma}+P_{a}, \quad \bar{H}_{a}=-\overrightarrow{\bar{P}}_{a}^{*} \cdot \vec{\sigma}+\bar{P}_{a}, \\
& P_{a}^{(*)}=\left(B^{(*)-}, \quad \bar{B}^{(*) 0}\right),
\end{aligned}
$$

where $\sigma^{i}$ are the Pauli matrices, and $a$ is the light flavor index, $P_{a}^{*}$ and $P_{a}$ annihilate the vector and pseudoscalar heavy mesons, respectively, and $\bar{P}_{a}^{(*)}$ annihilates the corresponding anti-particle. The leading effective Lagrangian describing the coupling of $Z_{b}$ states to the bottom and antibottom mesons can be written as that in Ref. [9],

$$
\begin{aligned}
\mathcal{L}_{Z_{b} H H} & =\frac{g_{0}}{2 \sqrt{2}} \operatorname{Tr}\left[Z_{a b}^{\dagger i} H_{a} \sigma^{i} \bar{H}_{b}\right]+\frac{g_{0}}{2 \sqrt{2}} \operatorname{Tr}\left[\left(Z^{T}\right)_{b a}^{i} \bar{H}_{b}^{\dagger} \sigma^{i} H_{a}^{\dagger}\right], \\
Z_{a b} & =\left(\begin{array}{cc}
\frac{1}{\sqrt{2}} Z_{b}^{(\prime) 0} & Z_{b}^{(\prime)-} \\
Z_{b}^{(\prime)+} & -\frac{1}{\sqrt{2}} Z_{b}^{(\prime) 0}
\end{array}\right)_{a b},
\end{aligned}
$$

where $Z_{a b}$ annihilates $Z_{a b}, Z_{a b}^{\dagger}$ creates $Z_{a b}$, and $g_{0}$ is defined in Eq. (4). The Lagrangian for the coupling of the $P$-wave quarkonia and the $B$ mesons reads [37]

$\mathcal{L}_{h_{b} H H}=\frac{g_{h}}{2} \operatorname{Tr}\left[h_{b}^{\dagger i} H_{a} \sigma^{i} \bar{H}_{a}\right]+$ H.c.

The chiral Lagrangian for the $B$ mesons and the $S$-wave quarkonia can be written as [12]

$$
\begin{aligned}
\mathcal{L}_{\mathrm{HH}} \mathrm{PT}= & g_{\pi} \operatorname{Tr}\left[\bar{H}_{a}^{\dagger} \sigma^{i} \bar{H}_{b}\right] A_{a b}^{i}-g_{\pi} \operatorname{Tr}\left[H_{a}^{\dagger} H_{b} \sigma^{i}\right] A_{b a}^{i} \\
& +\frac{1}{2} g_{1} \operatorname{Tr}\left[\Upsilon \bar{H}_{a}^{\dagger} H_{b}^{\dagger}\right] A_{a b}^{0} \\
& +\frac{1}{2} i g_{2} \operatorname{Tr}\left[\Upsilon \bar{H}_{a}^{\dagger} \sigma \cdot \overleftrightarrow{\partial} H_{a}^{\dagger}\right]+\text { H.c. },
\end{aligned}
$$


where $A \overleftrightarrow{\partial} B \equiv A(\partial B)-(\partial A) B, \Upsilon$ is the $2 \times 2$ matrix field defined as $\Upsilon=\vec{\Upsilon}(5 S) \cdot \vec{\sigma}+\eta_{b}(5 S)$, and $A_{\mu}$ is the axial vector pion current, which is given by

$$
\begin{aligned}
A_{\mu} & =\frac{i}{2}\left(\xi^{\dagger} \partial_{\mu} \xi-\xi \partial_{\mu} \xi^{\dagger}\right)=-\partial_{\mu} M / F_{\pi}+\cdots, \\
\xi & =e^{\frac{i M}{F_{\pi}}}, \quad M=\left(\begin{array}{cc}
\frac{1}{\sqrt{2}} \pi^{0} & \pi^{+} \\
\pi^{-} & -\frac{1}{\sqrt{2}} \pi^{0}
\end{array}\right), \\
F_{\pi} & =132 \mathrm{MeV} .
\end{aligned}
$$

We set $g_{\pi}=0.25$ as in $[9,42]$. Note that our convention is different from that in [9], because a factor of $\sqrt{2 M}$ has been absorbed into the field operator of the heavy meson in our convention [31], then our $g_{\pi}$ is half of the value which is used in Ref. [9]. The leading effective Lagrangian describing the $Z_{b} h_{b} \pi$ interactions reads

$\mathcal{L}_{Z_{b} h_{b} \pi}=g_{z} \varepsilon^{i j k} Z_{a b}^{i} h_{b}^{\dagger j} A_{a b}^{k}+$ H.c.,

which describes the direct decay of $Z_{b}^{(\prime)} \rightarrow h_{b}(m P) \pi$.

Finally, we come to the vertex describing decay of $\Upsilon(5 S)$ into $Z_{b}^{(\prime)} \pi$. The corresponding Lagrangian to the leading order of the chiral expansion is given by [9]

$\mathcal{L}_{\Upsilon Z_{b} \pi}=g_{\Upsilon} \Upsilon^{i}(5 S) Z_{b a}^{\dagger i} A_{a b}^{0}+$ H.c..

Similar to Ref. [9], we use the same coupling $g_{\Upsilon}, g_{z}$ for $Z_{b}$ and $Z_{b}^{\prime}$.

With the above effective Lagrangians and Eq. (8) as the propagator of $Z_{b}^{(\prime)}$, one can then write out the amplitudes for all the Feynman diagrams in Figs. 2 and 3. We treat the loop integrals as was done in Ref. [37]. We present the relevant one loop three-point functions in Appendix A, and give all the amplitudes of Figs. 2 and 3 in Appendix B. In the following we address several points before ending this section.

- As in Ref. [9], we assume that $Z_{b}$ only couples to $B \bar{B}^{*}$ while $Z_{b}^{\prime}$ only couples to $B^{*} \bar{B}^{*}$. We then find that there is a relative minus sign between $i \mathcal{M}_{3 a, 3 b, 3 c, 3 d}$ for $\Upsilon(5 S) \rightarrow Z_{b}^{+} \pi^{-} \rightarrow h_{b}(m P) \pi^{+} \pi^{-}$and those for $\Upsilon(5 S) \rightarrow Z_{b}^{\prime+} \pi^{-} \rightarrow h_{b}(m P) \pi^{+} \pi^{-}$. It should not be surprising to find this relative minus sign, since if one assumes $Z_{b}\left(Z_{b}^{\prime}\right)$ couples to $B^{*} \bar{B}^{*}\left(B \bar{B}^{*}\right)$ with the same strength as that of $Z_{b}\left(Z_{b}^{\prime}\right)$ couples to $B \bar{B}^{*}\left(B^{*} \bar{B}^{*}\right)$, one would find that the meson loop amplitudes would be suppressed in a heavy-quark spin symmetry world as noticed in [37].

- Assuming that $Z_{b}$ and $Z_{b}^{\prime}$ are spin partners of each other, we can use the same $Z$ for $Z_{b}$ and $Z_{b}^{\prime}$. In this way, we can reduce the number of free parameters in our fitting.

- We show the Feynman diagrams for non-resonant contributions to $\Upsilon(5 S) \rightarrow h_{b}(m P) \pi \pi$ in Fig. 4. Reference [43] shows that the non-resonant diagrams do not

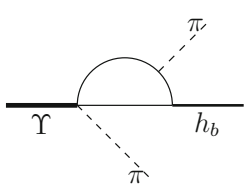

(a)

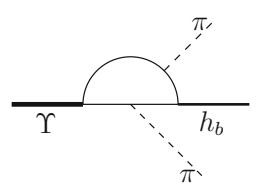

(b)

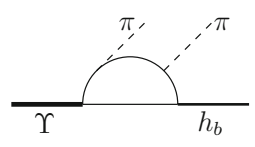

(c)
Fig. 4 Feynman diagrams for non-resonant processes $\Upsilon(5 S) \rightarrow$ $h_{b}(m P) \pi \pi$. Solid lines in the loop represent bottom and anti-bottom mesons

satisfy the two-cut condition near the $\Upsilon(5 S)$ region. Hence their contributions will not be enhanced by the kinematic singularity. We do not include their contributions in the present work, since they are suppressed by the heavy-quark spin symmetry. The experimental fits also find no significant non-resonant contributions [1,2].

\section{$4 \Upsilon(5 S)$ decays to $B^{(*)} \bar{B}^{(*)} \pi$}

In this section, we will study the decay $\Upsilon(5 S) \rightarrow B^{(*)} \bar{B}^{(*)} \pi$ in EFT. For the previous study one may refer to Ref. [12], where the $Z_{b}$ states are assumed to be molecules. Instead of fitting data directly, Ref. [12] constrains some parameters using data on $\Upsilon(5 S) \rightarrow B^{(*)} \bar{B}^{(*)}$, and it then calculates the differential distribution for $\Upsilon(5 S) \rightarrow B^{(*)} \bar{B}^{(*)} \pi$ as a function of the invariant mass of the $B^{(*)} \bar{B}^{(*)}$ pair. In this work, we give the amplitudes for $\Upsilon(5 S) \rightarrow B^{(*)} \bar{B}^{(*)} \pi$ in EFT and constrain parameters by fitting the data directly.

Similar to $\Upsilon(5 S) \rightarrow Z_{b}^{(\prime)} \pi \rightarrow h_{b}(m P) \pi \pi, Z_{b}$ states can be produced through both direct and indirect processes. The leading order Feynman diagrams for these two different production mechanisms are presented in Fig. 5. The Feynman diagrams for the non-resonant contributions are shown in Fig. 6. We give all the amplitudes for Figs. 5 and 6 in Appendix C.

\section{Numerical results}

With the amplitudes given in Appendices B and C, we do a combined fit to data on $\Upsilon(5 S) \rightarrow h_{b}(m P) \pi^{+} \pi^{-}[1,2]$ and $\Upsilon(5 S) \rightarrow B^{(*)} \bar{B}^{(*)} \pi$ [4]. Data on $\Upsilon(5 S) \rightarrow B^{(*)} \bar{B}^{*} \pi$ are nonvanishing below the $B^{(*)} \bar{B}^{(*)}$ thresholds, hence we have to convolve the invariant mass spectra with the detector resolution function. Data on $\Upsilon(5 S) \rightarrow h_{b}(m P) \pi^{+} \pi^{-}$are collected per $10 \mathrm{MeV}$, so the invariant mass spectra should be convolved with the detector resolution function and integrated over $10 \mathrm{MeV}$ histogram bin. The detector resolution is parameterized by a Gaussian function with energy resolution parameter $\sigma=5.2 \mathrm{MeV}$ for $\Upsilon(5 S) \rightarrow h_{b}(m P) \pi^{+} \pi^{-}[1]$ and $\sigma=6 \mathrm{MeV}$ for $\Upsilon(5 S) \rightarrow B^{(*)} \bar{B}^{(*)} \pi$ [4]. To compare different scenarios for the structure of $Z_{b}$ states, we do the fit with two alternative schemes: 
Fig. 5 Feynman diagrams for $\Upsilon(5 S) \rightarrow Z_{b}^{(\prime)} \pi \rightarrow B^{(*)} \bar{B}^{(*)} \pi$. Solid lines in the loop and in the final state represent bottom and anti-bottom mesons

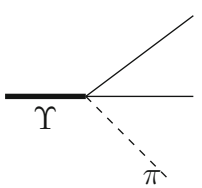

(a)

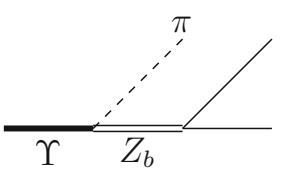

(a)

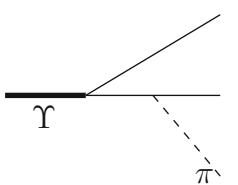

(b)
Fig. 6 Feynman diagrams of the non-resonant contribution to $\Upsilon(5 S) \rightarrow B^{(*)} \bar{B}^{(*)} \pi$. The solid lines represent the bottom and antibottom mesons

a. We assume that $Z_{b}$ states are pure molecular states, then we have to set $Z=0$ in the fit. In this way, only the diagrams in Figs. 3a, c, 5b, c, and 6 give nonvanishing amplitudes.

b. We assume that $Z_{b}$ states contain substantial compact components, i.e., a tetraquark component. It is shown in Ref. [22] that the production rate of a molecular state is proportional to its wave function squared at the origin $|\Psi(0)|^{2}$. Because the wave function of the molecular component in a loosely bound state spreads far out in space, $|\Psi(0)|^{2}$ is quite small, then the production rate of $Z_{b}^{(\prime)}$ through the molecular component will be suppressed. Therefore, we further assume that $Z_{b}^{(\prime)}$ is mainly produced through the compact component, and we set $g_{1}=g_{2}=0$ in the fitting. It is worth mentioning that Refs. [21,31] demonstrate that the production of a near threshold state (by which we mean a mixture of the compact component and molecular component) is driven by the compact component. On the other hand, the hadronic decays of $Z_{b}^{(\prime)}$ into $h_{b}(m P) \pi$ will mainly go through the molecular component. This can be found from the power counting analysis. We treat the binding momentum $\gamma$, the three-momentum of the bottom meson $p_{B}$ and the four momentum of the pion $p_{\pi}$ as small scales, i.e., they are all at the order of $\mathcal{O}(p)$. Note that in the non-relativistic effective field theory, the propagator of the heavy meson is at the order of $\mathcal{O}\left(p^{-2}\right)$, and the measure of the one

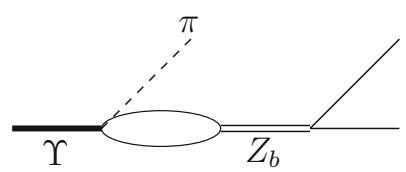

(b)

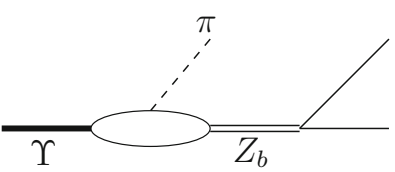

(c) loop integration is at the order of $\mathcal{O}\left(p^{5}\right)$. One can then find that Fig. $2 \mathrm{a}$ is at the order of $\mathcal{O}\left(p^{-1 / 2}\right)$, while Fig. $2 \mathrm{~b}$ is at the order of $\mathcal{O}\left(p^{0}\right)$. Thus, as a leading order study, we set $g_{z}=0$ and neglect the contribution from Fig. 2 b. Up to now, we have shown that while the production of $Z_{b}^{(\prime)}$ is driven by the compact component, its hadronic decays mainly go through the molecular component. It is interesting to note that similar features are adopted for $X$ (3872) in Ref. [21]. By setting $g_{1}=g_{2}=g_{z}=0$, one can find that only the diagrams Figs. 2a and 5a give nonvanishing amplitudes, and the number of the relevant free parameters in this scheme is the same as that in scheme (a) (see Table 1).

We then compare our fitting schemes with that used in Ref. [9]. Although scheme (b) and Ref. [9] use the same decay mechanism for $\Upsilon(5 S) \rightarrow Z_{b} \pi \rightarrow h_{b} \pi \pi$ as shown in Fig. 2a, there are some differences between them. The main difference is that Ref. [9] sets $Z=0$, while in scheme (b) we let $Z$ to be a free parameter which satisfies $0<Z<1$. As shown explicitly in Appendix B, the amplitude for Fig. 2a is zero by setting $Z=0$. Physically, by setting $Z=0$, one assumes the $Z_{b}$ states as pure molecular states which do not contain compact components, hence they cannot be produced through the compact components. Therefore, if one uses Fig. 2a to describe the decay mechanism of $\Upsilon(5 S) \rightarrow$ $Z_{b} \pi \rightarrow h_{b} \pi \pi$, one cannot set $Z=0$ as in Ref. [9]. The consistent treatment is to let $Z$ to be a free parameter which satisfies $0<Z<1$. On the other hand, if one assumes $Z_{b}^{(\prime)}$ to be a pure molecular state, i.e., $Z=0$, one should note that it can only be produced through an indirect process. Therefore, for the pure molecular scenario, one should use Fig. 3a, c, i.e., scheme (a), instead of Fig. 2a to describe the decay mechanism of $\Upsilon(5 S) \rightarrow Z_{b} \pi \rightarrow h_{b} \pi \pi$.

Now we come to discuss the applicability of EFT. In the decay $Z_{b}^{(\prime)} \rightarrow h_{b}(2 P) \pi$, the momentum of the pion is around $300-400 \mathrm{MeV}$ in the energy region of our con-

Table 1 Parameters for four fits

\begin{tabular}{llllllll}
\hline Fit & $g_{2} / g_{1}$ & $B$ & $B^{\prime}$ & $\Gamma_{Z_{b}}$ & $\Gamma_{Z_{b}^{\prime}}$ & $Z$ & $\chi^{2} /$ d.o.f. \\
\hline 1a & $0.049(15)$ & $0.11(12) \mathrm{eV}$ & $27(58) \mathrm{keV}$ & $2(1) \mathrm{keV}$ & $1.9(1.9) \mathrm{MeV}$ & 0 & $110 / 58$ \\
2a & $0.0017(69)$ & $12(21) \mathrm{keV}$ & $0.14(7) \mathrm{MeV}$ & $0.12(8) \mathrm{MeV}$ & $0.59(27) \mathrm{MeV}$ & 0 & $72 / 45$ \\
$\mathrm{lb}$ & - & $0.19(22) \mathrm{eV}$ & $1.6(1.8) \mathrm{eV}$ & $5.5(1.8) \mathrm{MeV}$ & $7.8(2.2) \mathrm{MeV}$ & $0.42(12)$ & $81 / 58$ \\
$\mathrm{2b}$ & - & $0.38(65) \mathrm{eV}$ & $0.51(86) \mathrm{eV}$ & $6.1(2.8) \mathrm{MeV}$ & $4.6(2.2) \mathrm{MeV}$ & $0.42(18)$ & $69 / 45$ \\
\hline
\end{tabular}



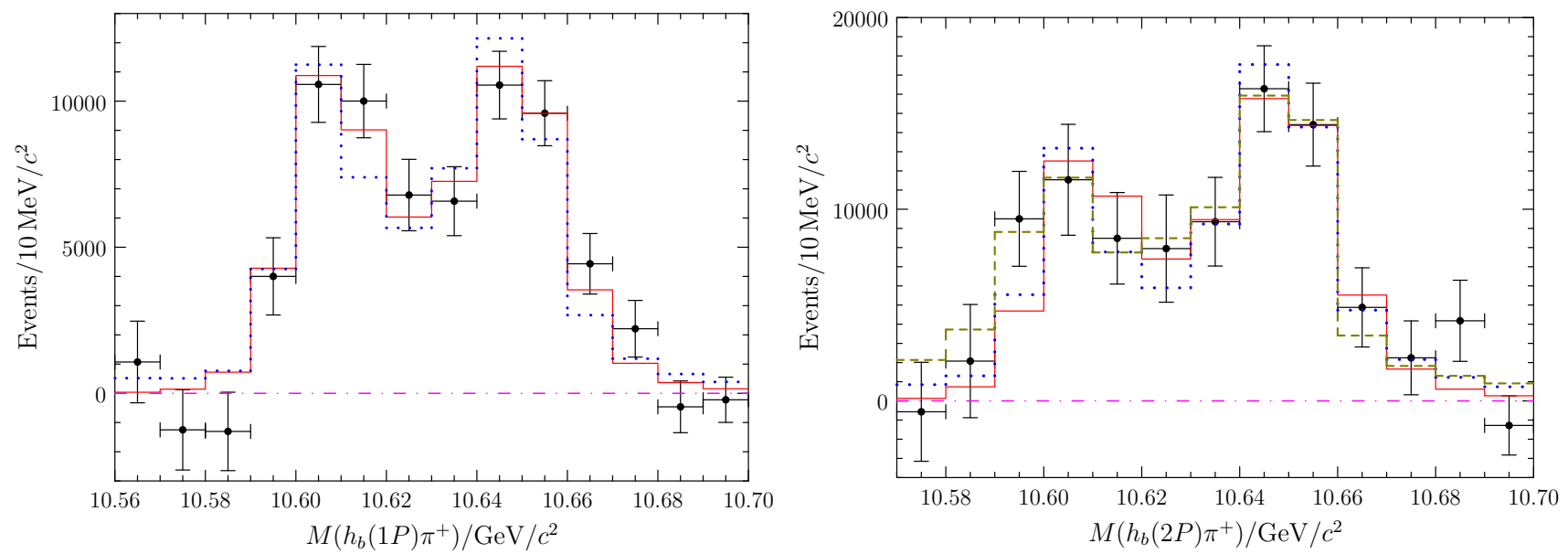

Fig. 7 Comparison of the invariant mass spectra of $h_{b}(1 P) \pi$ and $h_{b}(2 P) \pi$ in fit (1a), fit (2a), fit (1b) and the experiment. The dotted line is the result of fit (1a). The dashed line is the result of fit (2a). The solid line is the result of fit (1b). Data are from [1]
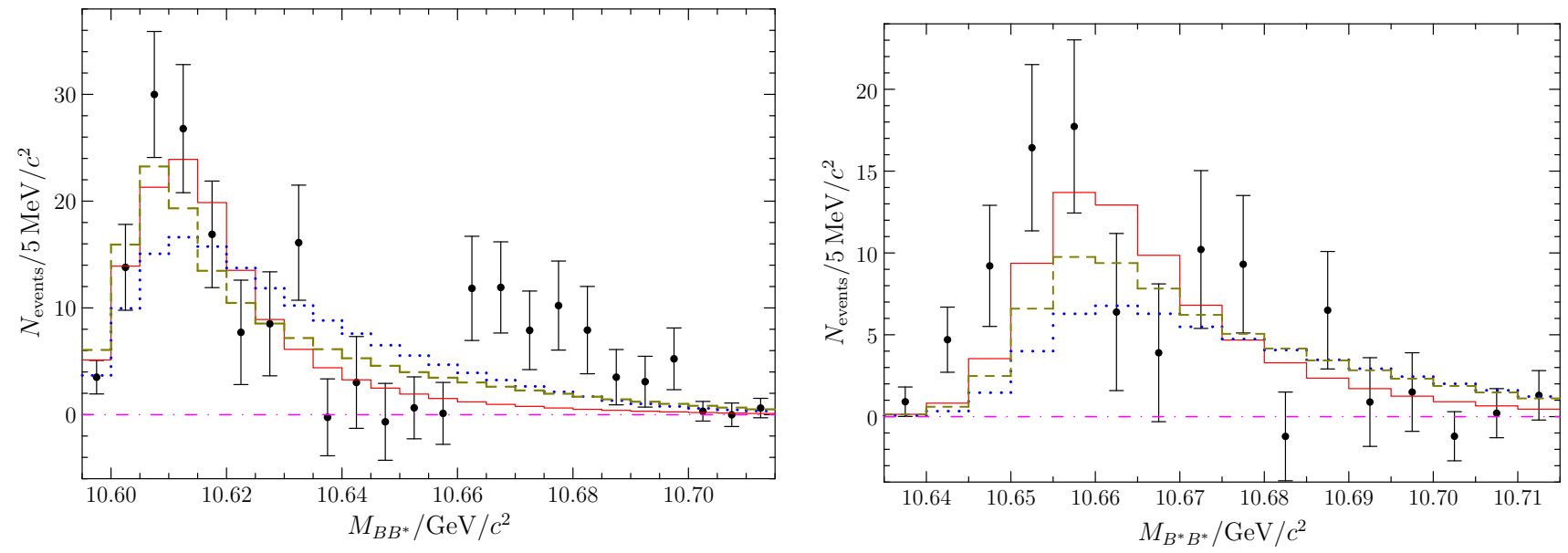

Fig. 8 Comparison of the invariant mass spectra of $B \bar{B}^{*}$ and $B^{*} \bar{B}^{*}$ in fit (1a), fit (2a), fit (1b) and the experiment. The dotted line is the result of fit (1a). The dashed line is the result of fit (2a). The solid line is the result of fit (1b). Data are from [4] and have had background subtracted

cern, hence the pion can be treated as soft, and one would expect the EFT expansion can converge fast enough. But in $Z_{b}^{(\prime)} \rightarrow h_{b}(1 P) \pi$, the momentum of the pion is relatively large and around 600-700 MeV. Based on naive dimensional analysis, Ref. [10] warns that the EFT expansion may not be good enough for decay $Z_{b}^{(\prime)} \rightarrow h_{b}(1 P) \pi$ due to the relatively large pion momentum. However, the results from the complete loop calculations can be more complex than the naive dimensional analysis. One may refer to Ref. [44] for an example. Generally, it is complex to study the convergence of the effective field theory, and reliable conclusions can only be reached once the complete higher loop contributions are available. Since such a kind of study is beyond the scope of this work, we take a more pragmatic approach with two options in the fit.

1. We use data sets of $\Upsilon(5 S) \rightarrow h_{b}(1 P, 2 P) \pi^{+} \pi^{-}$, $\Upsilon(5 S) \rightarrow B \bar{B}^{*} \pi$, and $\Upsilon(5 S) \rightarrow B^{*} \bar{B}^{*} \pi$ in our fit.
2. We use data sets of $\Upsilon(5 S) \rightarrow h_{b}(2 P) \pi^{+} \pi^{-}, \Upsilon(5 S) \rightarrow$ $B \bar{B}^{*} \pi$, and $\Upsilon(5 S) \rightarrow B^{*} \bar{B}^{*} \pi$ in our fit.

We choose an individual normalization factor for each final state in the fit. In this way, we need not to fix values of $g_{\Upsilon}$ and $g_{h}$. We present all the fitted parameters in Table. 1, and we show the fitting results of fit (1a), fit (2a) and fit (1b) in Figs. 7, 8. Note that the width $\Gamma$ in Table 1 is not the total width, but the width defined in Eq. (8).

We give some brief discussions as regards our fitting results as follows:

- It is found in the experimental fits that the relative phase between $Z_{b}$ and $Z_{b}^{\prime}$ in the $h_{b}(m P) \pi \pi$ channel is $180^{\circ}$ [1,2]. In fitting scheme (a), the relative minus sign between $i \mathcal{M}_{3 a, 3 c}$ for $Z_{b}$ and $Z_{b}^{\prime}$ can account for this relative phase. However, one cannot find such a relative phase in amplitudes which are used in scheme (b). 
In our fitting, we find that scheme (b) gives a good fit only if such a relative phase is included. This may be attributed to $g_{\Upsilon}$ [defined in Eq. (15)] which has a relative minus sign between $Z_{b}$ and $Z_{b}^{\prime}$. We note that a very recent paper, Ref. [45], proposed an explanation for this relative minus sign.

- From the fitting results of fit (1b) and fit (2b), one can find that the fitted parameters in fit (1b) and fit (2b) are close to each other. This indicates that the fitting results in scheme (b) are not sensitive to data on $\Upsilon(5 S) \rightarrow h_{b}(1 P) \pi^{+} \pi^{-}$. Whether this means that the effective field theory can be successfully applied in $\Upsilon(5 S) \rightarrow h_{b}(1 P) \pi^{+} \pi^{-}$needs to be further investigated. Nevertheless, our numerical results show that such a possibility exists. It is also interesting to find that in scheme (b) the fitted binding energy and the width of $Z_{b}$ are close to those of $Z_{b}^{\prime}$. This seems to be consistent with the heavy-quark spin symmetry.

- With all data sets, scheme (1b) gives much better fitting quality than scheme (1a). Unfortunately, if data on $\Upsilon(5 S) \rightarrow h_{b}(1 P) \pi^{+} \pi^{-}$are dropped, the two schemes give almost equal fitting qualities. In this sense, it seems too early to claim conclusively that $Z_{b}$ states contain substantial compact components. However, a substantial compact component in $Z_{b}^{(\prime)}$ can explain its large production rates in experiments. In contrast, a pure molecular state with the tiny binding energy as determined in scheme (a) is not likely to have large production rates in $\Upsilon(5 S)$ decays.

- The binding energies of the $Z_{b}$ states from the fit are generally very small. If we fix $B=0.1 \mathrm{MeV}$, which is the case for $X(3872)$, and $Z=0.4$ in fit (1b), we get a fitting quality $\chi^{2}=90$, which is still acceptable and better than fit (1a). The other fitting parameters are $B^{\prime}=0.23(14) \mathrm{MeV}, \Gamma_{Z_{b}}=6.5(9) \mathrm{MeV}$ and $\Gamma_{Z_{b}^{\prime}}=$ 5.6(9) MeV. This result also seems to be consistent with the heavy-quark symmetry.

- One can also analyze data on $\Upsilon(5 S) \rightarrow \Upsilon(n S) \pi^{+} \pi^{-}$in the EFT approach. However, different from $h_{b}(m P) \pi^{+} \pi^{-}$and $B^{(*)} \bar{B}^{(*)} \pi$, the non-resonant contribution in $\Upsilon(n S) \pi^{+} \pi^{-}$is significant. It is impossible to two-dimensional Dalitz distribution, which is beyond the scope of the present manuscript.

\section{Summary}

We have done a combined analysis of data on $\Upsilon(5 S) \rightarrow$ $h_{b}(1 P, 2 P) \pi^{+} \pi^{-}, \Upsilon(5 S) \rightarrow B \bar{B}^{*} \pi$ and $\Upsilon(5 S) \rightarrow$ $B^{*} \bar{B}^{*} \pi$ within EFT approach. With a combined analysis, we determine the resonance parameters of $Z_{b}$ states in two scenarios. In one scenario we assume that $Z_{b}$ states are pure molecular states, while in the other one we assume that $Z_{b}$ states contain compact components. It is found that by assuming that $Z_{b}$ states contain substantial compact components, one can have a better description of all data than by pure molecular assumption. By fitting the invariant mass spectra of $\Upsilon(5 S) \rightarrow h_{b}(1 P, 2 P) \pi^{+} \pi^{-}$and $\Upsilon(5 S) \rightarrow B^{(*)} \bar{B}^{(*)} \pi$, we determine that the probability of finding a compact component in $Z_{b}^{(\prime)}$ is about $40 \%$. It is also interesting to note that the probability of finding a compact component in $Z_{b}^{(\prime)}$ could be close to that in $X(3872)$, around 26-44\% $[46,47]$.

Acknowledgments We would like to thank Qiang Zhao for a careful reading of the manuscript and valuable comments and Feng-Kun Guo for very useful discussions. We would also like to thank Guang-Yi Tang for the helpful discussions on the Belle result. This work is supported, in part, by National Natural Science Foundation of China (Grant Nos. 11147022 and 11305137) and Doctoral Foundation of Xinjiang University (No. BS110104).

Open Access This article is distributed under the terms of the Creative Commons Attribution 4.0 International License (http://creativecomm ons.org/licenses/by/4.0/), which permits unrestricted use, distribution, and reproduction in any medium, provided you give appropriate credit to the original author(s) and the source, provide a link to the Creative Commons license, and indicate if changes were made.

Funded by SCOAP ${ }^{3}$.

\section{Appendix A: One loop three-point functions}

The three-point loop functions we will encounter are

$$
\begin{aligned}
I\left(m_{1}, m_{2}, m_{3}, q\right) & =(-i) \int \frac{\mathrm{d}^{d} \ell}{(2 \pi)^{d}} \times \frac{1}{\left(\ell^{0}-\frac{\vec{\ell}^{2}}{2 m_{1}}+i \epsilon\right)\left(\ell^{0}+b_{12}+\frac{\ell^{2}}{2 m_{2}}-i \epsilon\right)\left[\ell^{0}+b_{12}-b_{23}-\frac{(\vec{\ell}-\vec{q})^{2}}{2 m_{3}}+i \epsilon\right]} \\
& =\frac{\mu_{12} \mu_{23}}{2 \pi} \frac{1}{\sqrt{a}} \times\left[\tan ^{-1}\left(\frac{c^{\prime}-c}{2 \sqrt{a(c-i \epsilon)}}\right)+\tan ^{-1}\left(\frac{2 a+c-c^{\prime}}{2 \sqrt{a\left(c^{\prime}-a-i \epsilon\right)}}\right)\right],
\end{aligned}
$$

consider the interference with the non-resonant contribution correctly in one-dimensional analysis. To analyze data on $\Upsilon(5 S) \rightarrow \Upsilon(n S) \pi^{+} \pi^{-}$, one needs to fit the

$$
\begin{aligned}
I^{(1)}\left(m_{1}, m_{2}, m_{3}, q\right)= & \frac{\mu_{12} m_{3}}{2 \pi \vec{q}^{2}}\left(\sqrt{c-i \epsilon}-\sqrt{c^{\prime}-a-i \epsilon}\right) \\
& +\frac{m_{3}\left(c^{\prime}-c\right)}{2 \mu_{23} \vec{q}^{2}} I\left(m_{1}, m_{2}, m_{3}, q\right),
\end{aligned}
$$


where $\mu_{i j}=m_{i} m_{j} /\left(m_{i}+m_{j}\right)$ are the reduced masses, $b_{12}=$ $m_{1}+m_{2}-M, b_{23}=m_{2}+m_{3}+q^{0}-M$, and

$a=\left(\frac{\mu_{23}}{m_{3}}\right)^{2} \vec{q}^{2}, \quad c=2 \mu_{12} b_{12}$

$c^{\prime}=2 \mu_{23} b_{23}+\frac{\mu_{23}}{m_{3}} \vec{q}^{2}$.

$I^{(1)}\left(m_{1}, m_{2}, m_{3}, q\right)$ is defined as

$$
\begin{aligned}
& i \mathcal{M}_{3 d}=i 4 \sqrt{2} \frac{g g_{2} g_{z} g_{\pi}}{F_{\pi}^{2}} \\
& \times\left[\left(I^{(1)}\left(m_{B^{*}}, m_{B^{*}}, m_{B}, p\right)+I^{(1)}\left(m_{B^{*}}, m_{B}, m_{B^{*}}, p\right)\right) \vec{p}^{2} \delta^{k m}\right. \\
& \left.+\left(I^{(1)}\left(m_{B}, m_{B}, m_{B^{*}}, p\right)-I^{(1)}\left(m_{B^{*}}, m_{B}, m_{B^{*}}, p\right)\right) p^{k} p^{m}\right] \\
& \times \frac{\sqrt{Z}}{E+B+\tilde{\Sigma}(E)+i \Gamma_{Z_{b}} / 2} \times \varepsilon^{i j k} q^{i} \epsilon^{* j}\left(h_{b}\right) \epsilon^{m}(\Upsilon) .
\end{aligned}
$$

$q^{i} I^{(1)}\left(m_{1}, m_{2}, m_{3}, q\right)=(-i) \int \frac{\mathrm{d}^{d} \ell}{(2 \pi)^{d}} \times \frac{\ell^{i}}{\left(\ell^{0}-\frac{\vec{\ell}^{2}}{2 m_{1}}+i \epsilon\right)\left(\ell^{0}+b_{12}+\frac{\ell^{2}}{2 m_{2}}-i \epsilon\right)\left[\ell^{0}+b_{12}-b_{23}-\frac{(\vec{\ell}-\vec{q})^{2}}{2 m_{3}}+i \epsilon\right]}$.

For more details, one may refer to Ref. [37].

\section{Appendix B: Amplitudes for $\Upsilon(5 S) \rightarrow h_{b}(1 P, 2 P) \pi^{+} \pi^{-}$}

The amplitudes for $\Upsilon(5 S) \rightarrow Z_{b}^{+} \pi^{-} \rightarrow h_{b}(m P) \pi^{+} \pi^{-}$in

Figs. 2 and 3 read

$$
\begin{aligned}
i \mathcal{M}_{2 a}= & \frac{2 \sqrt{2 Z} g g_{\Upsilon} g_{\pi} g_{h} E_{\pi}}{F_{\pi}^{2}} \frac{1}{E+B+\tilde{\Sigma}(E)+i \Gamma_{Z_{b} / 2}} \\
& \times \varepsilon^{i j k} q^{i} \epsilon^{* j}\left(h_{b}\right) \epsilon^{k}(\Upsilon)\left[I\left(m_{B^{*}}, m_{B}, m_{B^{*}}, q\right)\right. \\
& \left.+I\left(m_{B}, m_{B^{*}}, m_{B^{*}}, q\right)\right] \\
i \mathcal{M}_{2 b}= & -i \frac{g_{\Upsilon} g_{z} E_{\pi}}{F_{\pi}^{2}} \frac{Z}{E+B+\tilde{\Sigma}(E)+i \Gamma_{Z_{b}} / 2} \\
& \times \varepsilon^{i j k} q^{i} \epsilon^{* j}\left(h_{b}\right) \epsilon^{k}(\Upsilon), \\
i \mathcal{M}_{3 a}= & -\frac{2 g^{2} g_{1} g_{\pi} g_{h} E_{\pi}}{F_{\pi}^{2}} \frac{\mu}{\pi}(-2 \mu E-i \epsilon)^{1 / 2} \\
& \times \frac{1}{E+B+\tilde{\Sigma}(E)+i \Gamma_{Z_{b} / 2}} \varepsilon^{i j k} q^{i} \epsilon^{* j}\left(h_{b}\right) \epsilon^{k}(\Upsilon) \\
& \times\left[I\left(m_{B^{*}}, m_{B}, m_{B^{*}}, q\right)+I\left(m_{B}, m_{B^{*}}, m_{B^{*}}, q\right)\right],
\end{aligned}
$$

$$
\begin{aligned}
i \mathcal{M}_{3 b}= & i \frac{\sqrt{2 Z}}{2 \pi} \frac{g g_{1} g_{z} E_{\pi}}{F_{\pi}^{2}} \mu(-2 \mu E-i \epsilon)^{1 / 2} \\
& \times \frac{1}{E+B+\tilde{\Sigma}(E)+i \Gamma_{Z_{b} / 2}} \varepsilon^{i j k} q^{i} \epsilon^{* j}\left(h_{b}\right) \epsilon^{k}(\Upsilon),
\end{aligned}
$$

$$
\begin{aligned}
& i \mathcal{M}_{3 c}=-16 \frac{g^{2} g_{2} g_{h} g_{\pi}^{2}}{F_{\pi}^{2}} \\
& \times\left[\left(I^{(1)}\left(m_{B^{*}}, m_{B^{*}}, m_{B}, p\right)+I^{(1)}\left(m_{B^{*}}, m_{B}, m_{B^{*}}, p\right)\right) \vec{p}^{2} \delta^{k m}\right. \\
& \left.+\left(I^{(1)}\left(m_{B}, m_{B}, m_{B^{*}}, p\right)-I^{(1)}\left(m_{B^{*}}, m_{B}, m_{B^{*}}, p\right)\right) p^{k} p^{m}\right] \\
& \times \frac{1}{E+B+\tilde{\Sigma}(E)+i \Gamma_{Z_{b}} / 2} \times \varepsilon^{i j k} q^{i} \epsilon^{* j}\left(h_{b}\right) \epsilon^{m}(\Upsilon) \\
& \times\left[I\left(m_{B^{*}}, m_{B}, m_{B^{*}}, q\right)+I\left(m_{B}, m_{B^{*}}, m_{B^{*}}, q\right)\right]
\end{aligned}
$$

$E$ is the energy defined relative to the $B B^{*}$ threshold. $B$ is the binding energy. $g$ is defined in Eq. (4). $E_{\pi}$ is the energy of $\pi^{-}, p$ is the three-momentum of the $\pi^{-}$, and $q$ is the three-momentum of the $\pi^{+} \cdot \mu=\frac{m_{B} m_{B^{*}}}{m_{B}+m_{B^{*}}}$ is the reduced mass. Note that the terms proportional to $p^{k} p^{m}$ in $\mathcal{M}_{3 c}$ and $\mathcal{M}_{3 d}$ will disappear in the heavy-quark limit, i.e., $m_{B}=$ $m_{B^{*}}$. This indicates that in the heavy-quark limit, the $\mathrm{D}$ wave decay of $\Upsilon(5 S) \rightarrow Z_{b} \pi$ is forbidden. We neglect the terms proportional to $p^{k} p^{m}$ in the fit, since they will be suppressed by the heavy-quark spin symmetry.

The amplitudes for $\Upsilon(5 S) \rightarrow Z_{b}^{\prime+} \pi^{-} \rightarrow h_{b}(m P) \pi^{+} \pi^{-}$ in Figs. 2 and 3 read

$$
\begin{aligned}
i \mathcal{M}_{2 a}= & \frac{2 \sqrt{2 Z} g^{\prime} g_{\Upsilon} g_{\pi} g_{h} E_{\pi}}{F_{\pi}^{2}} \frac{1}{E+B^{\prime}+\tilde{\Sigma}(E)+i \Gamma_{Z_{b}^{\prime}} / 2} \\
& \times \varepsilon^{i j k} q^{i} \epsilon^{* j}\left(h_{b}\right) \epsilon^{k}(\Upsilon) \times\left[I\left(m_{B^{*}}, m_{B^{*}}, m_{B^{*}}, q\right)\right. \\
& \left.+I\left(m_{B^{*}}, m_{B^{*}}, m_{B}, q\right)\right] \\
i \mathcal{M}_{2 b}= & -i \frac{g_{\Upsilon} g_{z} E_{\pi}}{F_{\pi}^{2}} \frac{Z}{E+B^{\prime}+\tilde{\Sigma}(E)+i \Gamma_{Z_{b}^{\prime}} / 2} \\
& \times \varepsilon^{i j k} q^{i} \epsilon^{* j}\left(h_{b}\right) \epsilon^{k}(\Upsilon),
\end{aligned}
$$

$$
\begin{aligned}
i \mathcal{M}_{3 a}= & \frac{2 g^{\prime 2} g_{1} g_{\pi} g_{h} E_{\pi}}{F_{\pi}^{2}} \frac{\mu}{\pi}(-2 \mu E-i \epsilon)^{1 / 2} \\
& \times \frac{1}{E+B^{\prime}+\tilde{\Sigma}(E)+i \Gamma_{Z_{b}^{\prime}} / 2} \times \varepsilon^{i j k} q^{i} \epsilon^{* j}\left(h_{b}\right) \epsilon^{k}(\Upsilon) \\
& \times\left[I\left(m_{B^{*}}, m_{B^{*}}, m_{B^{*}}, q\right)+I\left(m_{B^{*}}, m_{B^{*}}, m_{B}, q\right)\right]
\end{aligned}
$$

$$
\begin{aligned}
i \mathcal{M}_{3 b}= & -i \frac{\sqrt{2 Z}}{2 \pi} \frac{g^{\prime} g_{1} g_{z} E_{\pi}}{F_{\pi}^{2}} \mu(-2 \mu E-i \epsilon)^{1 / 2} \\
& \times \frac{1}{E+B^{\prime}+\tilde{\Sigma}(E)+i \Gamma_{Z_{b}^{\prime}} / 2} \varepsilon^{i j k} q^{i} \epsilon^{* j}\left(h_{b}\right) \epsilon^{k}(\Upsilon),
\end{aligned}
$$




$$
\begin{aligned}
& i \mathcal{M}_{3 c}=16 \frac{g^{\prime 2} g_{2} g_{h} g_{\pi}^{2}}{F_{\pi}^{2}} \\
& \times\left[\left(I^{(1)}\left(m_{B^{*}}, m_{B^{*}}, m_{B^{*}}, p\right)+I^{(1)}\left(m_{B}, m_{B^{*}}, m_{B^{*}}, p\right)\right) \vec{p}^{2} \delta^{k m}\right. \\
& \left.+\left(I^{(1)}\left(m_{B^{*}}, m_{B^{*}}, m_{B^{*}}, p\right)-I^{(1)}\left(m_{B}, m_{B^{*}}, m_{B^{*}}, p\right)\right) p^{k} p^{m}\right] \\
& \times \frac{1}{E+B^{\prime}+\tilde{\Sigma}(E)+i \Gamma_{Z_{b}^{\prime}} / 2} \times \varepsilon^{i j k} q^{i} \epsilon^{* j}\left(h_{b}\right) \epsilon^{m}(\Upsilon) \\
& \times\left[I\left(m_{B^{*}}, m_{B^{*}}, m_{B^{*}}, q\right)+I\left(m_{B^{*}}, m_{B^{*}}, m_{B}, q\right)\right], \\
& i \mathcal{M}{ }_{3 d}=-i 4 \sqrt{2} \frac{g^{\prime} g_{2} g_{z} g_{\pi}}{F_{\pi}^{2}} \\
& \times\left[\left(I^{(1)}\left(m_{B^{*}}, m_{B^{*}}, m_{B^{*}}, p\right)+I^{(1)}\left(m_{B}, m_{B^{*}}, m_{B^{*}}, p\right)\right) \vec{p}^{2} \delta^{k m}\right. \\
& \left.+\left(I^{(1)}\left(m_{B^{*}}, m_{B^{*}}, m_{B^{*}}, p\right)-I^{(1)}\left(m_{B}, m_{B^{*}}, m_{B^{*}}, p\right)\right) p^{k} p^{m}\right] \\
& \times \frac{\sqrt{Z}}{E+B^{\prime}+\tilde{\Sigma}(E)+i \Gamma_{Z_{b}^{\prime}} / 2} \times \varepsilon^{i j k} q^{i} \epsilon^{* j}\left(h_{b}\right) \epsilon^{m}(\Upsilon) .
\end{aligned}
$$

$E$ is the energy defined relative to the $B^{*} B^{*}$ threshold. $B^{\prime}$ is the binding energy. $g^{\prime}$ is the renormalized coupling constant which is defined in Eq. (4). We use $g^{\prime}$ here to distinguish from $g$, which is used in $\Upsilon(5 S) \rightarrow Z_{b}^{+} \pi^{-} \rightarrow h_{b}(m P) \pi^{+} \pi^{-}$, since they may be different due to different binding energies. $\mu$ is the reduced mass of the $B^{*} B^{*}$ system. Other notations are the same as that in $\Upsilon(5 S) \rightarrow Z_{b}^{+} \pi^{-} \rightarrow h_{b}(m P) \pi^{+} \pi^{-}$. We also neglect the terms proportional to $p^{k} p^{m}$ in the fit.

In the above, we have assumed that $Z_{b}$ only couples to $B \bar{B}^{*}$, while $Z_{b}^{\prime}$ only couples to $B^{*} \bar{B}^{*}$. We also assume that the probability of finding an elementary state in $Z_{b}$ and $Z_{b}^{\prime}$ is the same. In other words, we use the same $Z$ for $Z_{b}$ and $Z_{b}^{\prime}$.

\section{Appendix C: Amplitudes for $\Upsilon(5 S) \rightarrow B^{(*)} \bar{B}^{(*)} \pi$}

The amplitudes for $\Upsilon(5 S) \rightarrow B^{+} \bar{B}^{* 0} \pi$ in Figs. 5 and 6 read

$$
\begin{aligned}
& i \mathcal{M}_{5 a}=-\frac{g \Upsilon g E_{\pi}}{\sqrt{2} F_{\pi}} \frac{\sqrt{Z}}{E+B+\tilde{\Sigma}(E)+i \Gamma_{Z_{b}} / 2} \\
& \times \epsilon(\Upsilon) \cdot \epsilon^{*}\left(\bar{B}^{* 0}\right), \\
& i \mathcal{M}_{5 b}= g_{1} \frac{E_{\pi} \mu}{2 \pi F_{\pi}} \frac{g^{2} \sqrt{-2 \mu E-i \epsilon}}{E+B+\tilde{\Sigma}(E)+i \Gamma_{Z_{b}} / 2} \epsilon(\Upsilon) \cdot \epsilon^{*}\left(\bar{B}^{* 0}\right), \\
& i \mathcal{M}_{5 c}=4 \frac{g^{2} g_{2} g_{\pi}}{F_{\pi}} \epsilon^{* j}\left(\bar{B}^{* 0}\right) \epsilon^{m}(\Upsilon) \\
& \times\left[\left(I^{(1)}\left(m_{B^{*}}, m_{B^{*}}, m_{B}, q_{\pi}\right)+I^{(1)}\left(m_{B^{*}}, m_{B}, m_{B^{*}}, q_{\pi}\right)\right) \vec{q}_{\pi}^{2} \delta^{j m}\right. \\
&\left.+\left(I^{(1)}\left(m_{B}, m_{B}, m_{B^{*}}, q_{\pi}\right)-I^{(1)}\left(m_{B^{*}}, m_{B}, m_{B^{*}}, q_{\pi}\right)\right) q_{\pi}^{j} q_{\pi}^{m}\right] \\
& \quad \times \frac{1}{E+B+\tilde{\Sigma}^{\tilde{\Sigma}}(E)+i \Gamma_{Z_{b}} / 2}, \\
& i \mathcal{M}_{6 a}=g_{1} \frac{E_{\pi}}{F_{\pi}} \epsilon(\Upsilon) \cdot \epsilon^{*}\left(\bar{B}^{* 0}\right),
\end{aligned}
$$

$$
\begin{aligned}
i \mathcal{M}_{6 b}= & -4 \frac{g_{2} g_{\pi}}{F_{\pi}} \epsilon^{i}(\Upsilon) \epsilon^{* j}\left(\bar{B}^{* 0}\right) \\
& \times\left[\frac{1}{E_{\pi}+\Delta} p_{B}^{i} q_{\pi}^{j}-\frac{1}{E_{\pi}}\left(q_{\pi}^{i} p_{B}^{j}-q_{\pi} \cdot p_{B} \delta^{i j}\right)\right. \\
& \left.+\frac{1}{E_{\pi}-\Delta}\left(q_{\pi}^{i} p_{\bar{B}}^{j}+q_{\pi} \cdot p_{\bar{B}} \delta^{i j}-q_{\pi}^{j} p_{\bar{B}}^{i}\right)\right] .
\end{aligned}
$$

$E$ is the energy defined relative to the $B B^{*}$ threshold. $E_{\pi}$ is the pion energy, $q_{\pi}$ is the three-momentum of the pion. $\mu$ is the reduced mass of the $B B^{*}$ system. $p_{B}$ and $p_{\bar{B}}$ are the three-momenta of $B^{+}$and $\bar{B}^{* 0}$, respectively. $\Delta$ is the hyperfine splitting of the $B$ mesons.

The amplitudes for $\Upsilon(5 S) \rightarrow B^{*+} \bar{B}^{* 0} \pi$ in Fig. 5 and Fig. 6 read

$$
\begin{aligned}
& i \mathcal{M}_{5 a}=\frac{g \Upsilon g^{\prime} E_{\pi}}{\sqrt{2} F_{\pi}} \frac{i \sqrt{Z}}{E+B^{\prime}+\tilde{\Sigma}(E)+i \Gamma_{Z_{b}^{\prime}} / 2} \\
& \quad \times \varepsilon^{i m n} \epsilon^{i}(\Upsilon) \epsilon^{* m}\left(\bar{B}^{* 0}\right) \epsilon^{* n}\left(B^{*+}\right), \\
& i \mathcal{M}_{5 b}=\quad i g_{1} \frac{E_{\pi} \mu}{2 \pi F_{\pi}} \frac{g^{\prime 2} \sqrt{-2 \mu E-i \epsilon}}{E+B^{\prime}+\tilde{\Sigma}(E)+i \Gamma_{Z_{b}^{\prime}} / 2} \\
& \quad \times \varepsilon^{i m n} \epsilon^{i}(\Upsilon) \epsilon^{* m}\left(\bar{B}^{* 0}\right) \epsilon^{* n}\left(B^{*+}\right), \\
& i \mathcal{M}_{5 c}=i 4 \frac{g^{\prime 2} g_{2} g_{\pi}}{F_{\pi}} \\
& \times\left[\left(I^{(1)}\left(m_{B^{*}}, m_{B^{*}}, m_{B^{*}}, q_{\pi}\right)+I^{(1)}\left(m_{B}, m_{B^{*}}, m_{B^{*}}, q_{\pi}\right)\right) \vec{q}_{\pi}^{2} \delta^{k m}\right. \\
& \left.+\left(I^{(1)}\left(m_{B^{*}}, m_{B^{*}}, m_{B^{*}}, q_{\pi}\right)-I^{(1)}\left(m_{B}, m_{B^{*}}, m_{B^{*}}, q_{\pi}\right)\right) q_{\pi}^{k} q_{\pi}^{m}\right] \\
& \quad \times \frac{1}{E+B^{\prime}+\tilde{\Sigma}(E)+i \Gamma_{Z_{b}^{\prime} / 2} \times \varepsilon^{k j i} \epsilon^{m}(\Upsilon) \epsilon^{* j}\left(\bar{B}^{* 0}\right) \epsilon^{* i}\left(B^{*+}\right),}
\end{aligned}
$$

$$
\begin{aligned}
i \mathcal{M}_{6 a}= & -i g_{1} \frac{E_{\pi}}{F_{\pi}} \varepsilon^{i m n} \epsilon^{i}(\Upsilon) \epsilon^{* m}\left(B^{*+}\right) \epsilon^{* n}\left(\bar{B}^{* 0}\right), \\
i \mathcal{M}_{6 b}= & -i 4 \frac{g_{2} g_{\pi}}{F_{\pi}} \epsilon^{i}(\Upsilon) \epsilon^{* j}\left(B^{*+}\right) \epsilon^{* k}\left(\bar{B}^{* 0}\right) \\
& \times\left[\frac{1}{E_{\pi}+\Delta}\left(-\varepsilon^{i j m} p_{B}^{m} q_{\pi}^{k}+\varepsilon^{i k m} p_{\bar{B}}^{m} q_{\pi}^{j}\right)\right. \\
& +\frac{1}{E_{\pi}}\left(\varepsilon^{j n m} \delta^{i k} p_{\bar{B}}^{m} p_{B}^{n}-\varepsilon^{k m n} \delta^{i j} p_{\bar{B}}^{m} p_{B}^{n}\right. \\
& \left.\left.+\varepsilon^{m j k} q_{\pi}^{m} q_{\pi}^{i}-\varepsilon^{m k i} q_{\pi}^{m} p_{B}^{j}+\varepsilon^{m j i} q_{\pi}^{m} p_{\bar{B}}^{k}\right)\right] .
\end{aligned}
$$

$E$ is the energy defined relative to the $B^{*} B^{*}$ threshold. $E_{\pi}$ is the pion energy, $q_{\pi}$ is the three-momentum of the pion. $\mu$ is the reduced mass of the $B^{*} B^{*}$ system. $p_{B}$ and $p_{\bar{B}}$ are the three-momenta of $B^{*+}$ and $\bar{B}^{* 0}$, respectively. $\Delta$ is the hyperfine splitting of the $B$ mesons. 


\section{References}

1. I. Adachi [Belle Collaboration], arXiv:1105.4583 [hep-ex]

2. A. Bondar et al. [Belle Collaboration], Phys. Rev. Lett. 108, 122001 (2012). arXiv:1110.2251 [hep-ex]

3. K.A. Olive et al., [Particle Data Group Collaboration]. Chin. Phys. C 38, 090001 (2014)

4. I. Adachi et al. [Belle Collaboration], arXiv:1209.6450 [hep-ex]

5. A.E. Bondar, A. Garmash, A.I. Milstein, R. Mizuk, M.B. Voloshin, Phys. Rev. D 84, 054010 (2011). arXiv: 1105.4473 [hep-ph]

6. Y. Dong, A. Faessler, T. Gutsche, V.E. Lyubovitskij, J. Phys. G 40, 015002 (2013). arXiv:1203.1894 [hep-ph]

7. X. Li, M.B. Voloshin, Phys. Rev. D 86, 077502 (2012). arXiv:1207.2425 [hep-ph]

8. G. Li, F. -1. Shao, C.-W. Zhao, Q. Zhao, Phys. Rev. D 87(3), 034020 (2013). arXiv:1212.3784 [hep-ph]

9. M. Cleven, F.-K. Guo, C. Hanhart, U.-G. Meissner, Eur. Phys. J. A 47, $120(2011)$

10. M. Cleven, Q. Wang, F. -K. Guo, C. Hanhart, U.-G. Meissner, Q. Zhao, Phys. Rev. D 87(7), 074006 (2013). arXiv:1301.6461 [hep-ph]

11. T. Mehen, J.W. Powell, Phys. Rev. D 84, 114013 (2011). [arXiv:1109.3479 [hep-ph]

12. T. Mehen, J. Powell, Phys. Rev. D 88(3), 034017 (2013). arXiv: 1306.5459 [hep-ph]

13. A. Ali, C. Hambrock, W. Wang, Phys. Rev. D 85, 054011 (2012). arXiv: 1110.1333 [hep-ph]

14. E.S. Swanson, arXiv:1409.3291 [hep-ph]

15. D.V. Bugg, Europhys. Lett. 96, 11002 (2011). arXiv:1105.5492 [hep-ph]

16. M. Ablikim et al. [BESIII Collaboration], Phys. Rev. Lett. 110, 252001 (2013). arXiv:1303.5949 [hep-ex]

17. Z.Q. Liu et al. [Belle Collaboration], Phys. Rev. Lett. 110, 252002 (2013). arXiv:1304.0121 [hep-ex]

18. T. Xiao, S. Dobbs, A. Tomaradze, K.K. Seth, Phys. Lett. B 727, 366 (2013). arXiv:1304.3036 [hep-ex]

19. M. Ablikim et al. [BESIII Collaboration], Phys. Rev. Lett. 111(24), 242001 (2013). arXiv:1309.1896 [hep-ex]

20. M. Ablikim et al. [BESIII Collaboration], Phys. Rev. Lett. 112(2), 022001 (2014). arXiv:1310.1163 [hep-ex]

21. C. Meng, Y.J. Gao, K.T. Chao, Phys. Rev. D 87(7), 074035 (2013). arXiv:hep-ph/0506222

22. M. Suzuki, Phys. Rev. D 72, 114013 (2005). arXiv:hep-ph/0508258

23. R. Aaij et al. [LHCb Collaboration], Nucl. Phys. B 886, 665 (2014) arXiv: 1404.0275 [hep-ex]

24. Y. Dong, A. Faessler, T. Gutsche, V.E. Lyubovitskij, J. Phys. G 38, 015001 (2011). arXiv:0909.0380 [hep-ph]
25. F.K. Guo, C. Hanhart, Y.S. Kalashnikova, U.-G. Meissner, A.V. Nefediev, arXiv:1410.6712 [hep-ph]

26. T. Mehen, R. Springer, Phys. Rev. D 83, 094009 (2011). arXiv:1101.5175 [hep-ph]

27. M.T. AlFiky, F. Gabbiani, A.A. Petrov, Phys. Lett. B 640, 238 (2006). arXiv:hep-ph/0506141

28. G. Aad et al. [ATLAS Collaboration], Phys. Lett. B 740, 199 (2015). arXiv: 1410.4409 [hep-ex]

29. X.H. He et al. [Belle Collaboration], Phys. Rev. Lett. 113(14), 142001 (2014). arXiv: 1408.0504 [hep-ex]

30. M. Karliner, J.L. Rosner, Phys. Rev. D 91(1), 014014 (2015). arXiv: 1410.7729 [hep-ph]

31. G.Y. Chen, W.S. Huo, Q. Zhao, Chin. Phys. C 39(9), 093101 (2015). arXiv:1309.2859 [hep-ph]

32. C. Meng, J.J. Sanz-Cillero, M. Shi, D.L. Yao, H.Q. Zheng, Phys. Rev. D 92(3), 034020 (2015). doi:10.1103/PhysRevD.92.034020. arXiv:1411.3106 [hep-ph]

33. S. Weinberg, Phys. Rev. 130, 776 (1963)

34. S. Weinberg, Phys. Rev. 137, B672 (1965)

35. D.B. Kaplan, M.J. Savage, M.B. Wise, Phys. Lett. B 424, 390 (1998). arXiv:nucl-th/9801034

36. D.B. Kaplan, M.J. Savage, M. B. Wise, Nucl. Phys. B 534, 329 (1998). arXiv:nucl-th/9802075

37. F.-K. Guo, C. Hanhart, G. Li, U.-G. Meissner, Q. Zhao, Phys. Rev. D 83, 034013 (2011). arXiv:1008.3632 [hep-ph]

38. F.K. Guo, C. Hanhart, U.G. Meissner, Phys. Rev. Lett. 103, 082003 (2009). [Erratum-ibid. 104, 109901 (2010)]. arXiv:0907.0521 [hep-ph]

39. F.K. Guo, C. Hanhart, G. Li, U.G. Meissner, Q. Zhao, Phys. Rev. D 82, 034025 (2010). arXiv:1002.2712 [hep-ph]

40. J. Hu, T. Mehen, Phys. Rev. D 73, 054003 (2006). arXiv:hep-ph/0511321

41. S. Fleming, T. Mehen, Phys. Rev. D 78, 094019 (2008). arXiv:0807.2674 [hep-ph]

42. X.-Q. Li, F. Su, Y.-D. Yang, Phys. Rev. D 83, 054019 (2011). arXiv:1011.0269 [hep-ph]

43. Q. Wang, C. Hanhart, Q. Zhao, Phys. Lett. B 725(1-3), 106 (2013). arXiv:1305.1997 [hep-ph]

44. S. Fleming, T. Mehen, I.W. Stewart, Nucl. Phys. A 677, 313 (2000). arXiv:nucl-th/9911001

45. A. Ali, L. Maiani, A.D. Polosa, V. Riquer, Phys. Rev. D 91(1), 017502 (2015). arXiv:1412.2049 [hep-ph]

46. C. Meng, H. Han, K.-T. Chao, arXiv:1304.6710 [hep-ph]

47. M. Cardoso, G. Rupp, E. van Beveren, arXiv:1411.1654 [hep-ph] 